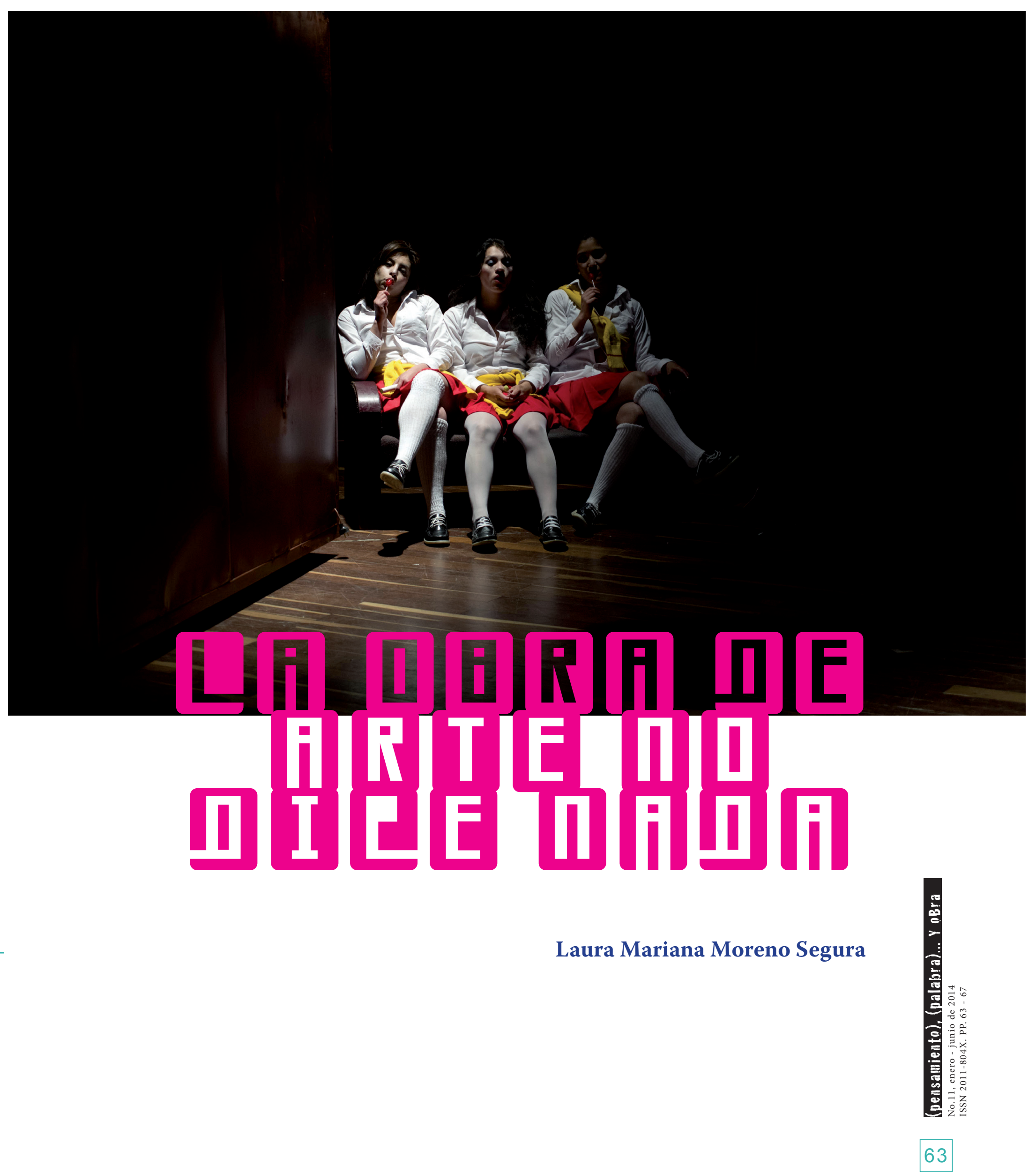




\section{LA OBRA DE ARTE NO DICE NADA ${ }^{1}$}

\section{Resumen}

En el siguiente trabajo busco explicar por qué las experiencias estéticas no pueden definirse por completo por medio del lenguaje. Parto de tal premisa teniendo en cuenta la teoría expuesta por el pensador Ludwig Wittgenstein, especialmente sobre las limitaciones del lenguaje. Para abordar este problema de una mejor manera, me valdré de las obras de Magritte y Duchamp, poniendo además en tela de juicio qué puede o no considerarse como una obra de arte. Finalmente haré un acercamiento sobre esta cuestión a la luz de Foucault y, principalmente, Gadamer.

Palabras clave: Lenguaje, límites, interpretación, experiencia estética, sentido.

\section{THE WORK OF ART DOES NOT SAY ANYTHING}

Abstract: In the following paper I'll try to explain how aesthetic experiences cannot be utterly defined through language. I'll start from this point, supporting my arguments on the theory of the so called second Wittgenstein regarding language's limitations. So as to address the issue in a better way and taking the works of Magritte and Duchamp as referents, I will establish some connections between art and philosophy, thus raising the question of what may or may not be considered as a work of art. Finally, we shall approach the issue under the light shed by Foucault's and mainly Gadamer's theories.

Key words: Language, limits, interpretation, aesthetic experience, sense.

\section{A OBRA DE ARTE NÃO DIZ NADA}

Resumo: No seguinte trabalho procuro explicar por que as experiências estéticas não podem definir-se inteiramente por meio da linguagem. Parto de esta premissa tendo em conta a teoria exposta pelo segundo Wittgenstein sobre as limitações da linguagem. Para abordar este problema de um melhor jeito, utilizarei as obras de Magritte e Duchamp pondo alem em tela de juízo que pode ou não considerar-se como uma obra de arte. Finalmente ficarei perto da questão nas luzes de Focault e principalmente Gadamer.

Palavras chave: linguagem, limites, interpretação, experiência estética, sentido.

\footnotetext{
${ }^{1}$ Ponencia presentada en el primer coloquio interno de la Licenciatura en Filosofía de la Universidad Pedagógica Nacional realizado del 22 al 26 de abril del año 2013.
} 
Había llegado a ese punto de emoción en el que se encuentran las sensaciones celestes dadas por las Bellas Artes y los sentimientos apasionados. Saliendo de Santa Croce, me latía el corazón, la vida estaba agotada en mí, andaba con miedo a caerme.

STHENDAL(1998)

Cuando nos encontramos delante de una obra de arte, tratamos de urdir siempre una significación que de algún modo podamos reducir a propiedades de nuestro interés, ya sean de carácter figurativo, pictórico, narrativo, histórico o incluso referentes a su composición. Podemos asegurar, entonces, que una obra de arte es bella, ${ }^{2}$ empero, cuando hacemos este tipo de afirmaciones generalmente estamos preparados para argumentar aquello que hemos dicho refiriéndonos a las cualidades que en la obra hemos observado, las cuales, hasta cierto punto, pueden calificarse como propias, esto es, cargadas de subjetividad.

El problema de analizar una obra de arte de esta manera radica en las limitaciones del lenguaje con el cual describimos, valoramos o juzgamos la obra de arte, tratando de hacer observaciones únicas e inequívocas al respecto. Las experiencias estéticas no alcanzan claridad por medio de su configuración con palabras, es decir, lo que estamos en capacidad de sentir frente a la obra se halla fuera del lenguaje, aun cuando estas palabras surgen de una necesidad primordial, una experimentación sensible del observador frente a la obra. ${ }^{3}$ En este sentido la obra es ajena al discurso, y por tanto lo es al sujeto que solo puede expresarse a través del mismo, como seguramente le ocurrió al pobre Sthendal (ver epígrafe) en su paseo por Santa Croce, de ahí que la obra no pueda reducirse ni acabarse ante infinitas interpretaciones.

Es claro que podemos otorgarle unas cualidades a la obra de arte y que al referirnos a una en especial no podemos ignorar el tiempo y el espacio en el cual fue creada para que fuese esta obra y no otra. Sin embargo, en La actualidad de lo bello, Gadamer (1997) nos muestra claramente que la obra per se está destinada desde un principio a su utilización, es decir, ella es independiente del artista, de las significaciones que se le den, e incluso de todos los elementos figurativos que de ella puedan extraerse. Así, uno de los problemas un tanto paradójicos que este autor plantea es el de la palabra: "Nunca debemos subestimar lo que una palabra pueda decirnos. La palabra es un anticipo del pensar ya antes que nosotros" (Gadamer, 1997). Esto quiere decir que aunque quisiéramos dar una última significación a la obra, la palabra nos permite discernir ciertas cualidades del objeto, pero no lo define para nuestros propósitos.

Tomemos como ejemplo la obra de Marcel Duchamp (1917), obviando el problema de lo que es y no es arte, qué debe y no debe estar en un museo. Podemos asegurar unas

${ }^{2}$ En esta frase, el concepto de belleza remite a una definición limitada que básicamente obedece a la aceptación social.

${ }^{3}$ Cuando me refiero aquí a una experimentación sensible, considero lo que antecede al lenguaje, hablo entonces de nuestras primeras relaciones con los objetos. intenciones trasgresoras en la obra de este artista que podrían incluso llegar a ser contradictorias y polémicas entre sí. El hecho es que, desde entonces, el impacto de su obra no ha podido ser determinado por las influencias históricas, de vanguardia, ${ }^{4}$ la firma del autor o la posición, simetría y composición en la que, por ejemplo, ha puesto un orinal dentro de un museo. Incluso, puede que si tuviésemos la oportunidad de hacer una pesquisa detallada (¿Qué es lo que significa este orinal?) basándonos en lo que buscaba el artista, las respuestas difirieran de las opiniones de muchos críticos e historiadores de arte, quienes aseguran que, en efecto, este suceso ha sido uno de los grandes cambios en la historia del arte moderno occidental y continúa asumiendo el lugar de una obra digna de encontrarse en un museo.

En este caso hemos visto cómo la obra de arte ha escapado al sentido que quiso expresar su creador, la obra es ajena a él, y con mayor razón, conseguirá serlo para nosotros mismos, los observadores; razón por la cual no podemos negar el poder que la obra $-\mathrm{y}$ no el artista - tiene sobre nosotros para lograr causar una experiencia estética genuina. La obra es bella en sí misma, esto significa que no se puede escindir de su propia belleza, que no puedo pensar en lo bello sin referirme a la obra de arte, sin conocer $^{5}$ la obra de arte.

Ahora bien, si partimos del problema del cómo conocemos y sobre todo qué conocemos de la obra de arte, de nuevo retornaremos al lenguaje. Empero, este conocimiento se adquiere de una experiencia propia que puede ser comprendida desde lo sensible, la apropiación teórica, e incluso desde los ideales de belleza preestablecidos culturalmente, puesto que todo lo que podemos examinar de la obra acaba en lo que ella nos muestra: lo que intentemos descifrar de la obra es relativo, no absoluto. La obra puede tener desde ciertas perspectivas un carácter informativo, de ruptura con parámetros artísticos o formalistas, pero lo que conocemos de la obra no se puede expresar de una forma completa ${ }^{6}$ en el lenguaje.

\footnotetext{
${ }^{4}$ Muchos historiadores de arte aseguran que, en sus inicios, Marcel Duchamp experimenta con todos los estilos de su época, hace pinturas impresionistas, fauvistas, pertenecientes al romanticismo, el simbolismo, el cubismo y el futurismo.

${ }^{5}$ No afirmaré la posibilidad de llegar a un conocimiento específico. Sin embargo, no niego nuestra capacidad para llegar a conocer algo, considerando antes la gran limitación que el lenguaje tiene sobre nuestros pensamientos y asociaciones con entidades simples.

${ }^{6}$ El uso de las palabras nos permite dar una significación y asumir que lo que sigue al objeto es compartido con otros sujetos, a pesar de esto, el uso del lenguaje es útil pero no nos da la posibilidad de conocer lo que el otro asume, ni lo que es el objeto en sí.
} 
Sabemos, de acuerdo con el primer Wittgenstein (2010), que solo percibimos hechos y estados de cosas. ${ }^{7}$ Antes bien, si evaluamos el uso del lenguaje, al cual el segundo Wittgenstein (1997) llama lenguaje ordinario, observamos que este se refiere principalmente a las formas proposicionales, lo cual hasta el momento no contradice lo que había dicho el filósofo en el Tractatus. Las formas proposicionales nos permiten conocer los fenómenos hasta cierto punto, no en su totalidad, aun si su estructura lógica es correcta. Dicho textualmente por Wittgenstein esto es: "Sólo podemos llegar a un análisis correcto mediante lo que se podría llamar la investigación lógica de los fenómenos mismos, en un cierto sentido a posteriori, y no mediante conjeturas sobre posibilidades a priori" (2010). No niego aquí la utilidad de las estructuras lógicas, pero hay que decir que estas no nos dan un conocimiento total del mundo. Lo anterior nos lleva a considerar que nuestra pretensión de significación dada a los conceptos es múltiple y susceptible de ambigüedad en la interpretación de los mismos.

Aunque Wittgenstein no se refiera precisamente a las experiencias estéticas, la investigación de tales fenómenos con relación al tema que nos interesa nos conduce a la imposibilidad de conformación de estructuras lógicas que permitan un conocimiento último de la obra, puesto que deben ir más allá de la libre organización de las palabras y entrar al análisis de aspectos que se quedan fuera de nuestro lenguaje:

Ahora bien, si intentamos obtener un análisis real, encontramos formas lógicas que se parecen muy poco a las normas del lenguaje ordinario. Encontramos las formas del espacio y el tiempo, con toda la diversidad de objetos espaciales y temporales, como colores, sonidos etc., con sus gradaciones, transiciones continuas, y combinaciones en diversas proporciones, todo lo cual no podemos aprehender a través de nuestros medios ordinarios de expresión (Wittgenstein, 1997).

Podríamos pensar que cuando Wittgenstein habla de la imposibilidad de aprehensión del significado por medio del lenguaje ordinario, guarda una estrecha relación con lo que entendemos como juicios estéticos, a lo que se sigue que la experiencia directa con el objeto, nuestra experiencia, no se haga letras en el discurso. Podemos hablar desde lo que ya se ha dicho, nuestros enunciados son ínfimos comparados con lo que sucedería sin una asociación a objetos ya clasificados en nuestra mente.

Afirmaré ahora, siguiendo la tesis de este autor, que mediante un análisis lógico no podemos señalar una relación de parámetros dentro de una sola propiedad que no excluya otra posibilidad de la misma. Wittgenstein se refiere aquí a los enunciados suplementarios, ${ }^{8}$ y dice que el análisis de un producto lógico finaliza cuando ya no existen más cualidades que a este puedan

${ }^{7}$ Para Wittgenstein los estados de cosas son la configuración objetos simples y estos a su vez no pueden ser definidos por ser la esencia del mundo, lo que permite la estabilidad del mismo.

${ }^{8}$ Un enunciado suplementario es aquel que niega la posibilidad de otro en el mismo espacio tiempo; tomemos en cuenta el siguiente ejemplo: El gato es gris. Este sería el enunciado suplementario de otros enunciados como: El gato es blanco o El gato es verde. atribuirse, tendrían validez entonces dichos análisis en nuestras apreciaciones de la obra, dándole de este modo una forma más concreta y por consiguiente menos apresurada.

Cabe resaltar que solo aquellos enunciados que son de carácter epistemológico podrían aceptarse como verdaderos. Así, por ejemplo, si decimos con fines meramente descriptivos que en la obra de Van Gogh titulada Girasoles (1888) las flores que se observan en el cuadro son sin duda girasoles, este es un enunciado suplementario que nos quita la posibilidad de afirmar que las flores del cuadro son rosas o margaritas. Por otro lado, si decimos, refiriéndonos a la misma obra, que el color de los girasoles refleja la personalidad del artista, este enunciado suplementario no necesariamente es verdadero pues nos quita la posibilidad de negar tal afirmación o darle una cualidad diferente al objeto, en este caso al color de los girasoles, pero conviene advertir que tal observación se aleja del plano descriptivo y se acerca más a lo que aceptamos como un juicio estético.

Ahora, se comprende por qué el utilizar la lógica como herramienta en la construcción de juicios estéticos resulta insuficiente. Si por medio de esta se nos revelara alguna verdad sobre la belleza de la obra de arte, la estética no sería tan problemática y bastaría tan solo con un análisis correcto $a$ posteriori.

La obra no nos dice nada, solo nos muestra algo que no puede ser tarea del lenguaje, pues este precisamente limita lo que pensamos y la forma en la que pensamos, mientras que una obra de arte, respetando la concepción de arte tan inveterada en nuestra cultura occidental y de acuerdo con Gadamer (1998), reclama una validez propia. Podemos pensar en los elementos que conforman la obra misma y nuestras observaciones de ella dentro de un sinfín de variables que hubiesen podido cambiar nuestras apreciaciones. Así, en lo único que podemos pensar es en preacuerdos con nuestra cultura, convenciones con nuestros pensamientos, pero aún más en aquello que configura esa manera de pensar, en lo que podrían llamarse las limitaciones del lenguaje.

Existe, pues, un sentimiento desesperado por tener la última palabra en lo que se refiere ala crítica de la obra, sentimiento que podemos intentar describir, pero no conoceremos completamente. Es ahí donde el arte juega un papel determinante en el lenguaje, porque nos muestra lo que no puede decirse con él, pero no nos está diciendo nada que podamos comprender de la manera arquetípica en la cual aprehendemos el conocimiento y, en últimas, lo que se nos dice de este. 
Para ejemplificar mejor esta idea, tomaré ahora como ejemplo la obra de Magritte de Ceci n'est pas une pipe, puesto que en la obra se confronta lo que no se puede decir, sino solo mostrar. Aunque Foucault, en su ensayo sobre la obra de Magritte, elimine el principio de contradicción dentro de ella, la dicotomía existente entre el lenguaje discursivo y el lenguaje del arte ${ }^{9}$ se hace presente de nuevo: "Lo extraño de esa figura no es la contradicción entre la imagen y el texto. Por una simple razón: tan solo puede haber contradicción entre dos enunciados, o en el interior de un mismo enunciado" (Foucault, 1989). Mas no se trata solamente de suprimir la demostración que podríamos pensar se halla inmersa en la obra. La idea de la pipa sigue latente aun después de darle una descripción del cuadro al lector, en el cual se encuentra ilustrada, en la parte superior, una pipa sobre la frase Ceci n'est pas une pipe (esto no es una pipa); me sirvo de tal ejemplo para confirmar que finalmente también esto reduciría nuestras posibilidades de interpretación, teniendo en cuenta los dos principios de contradicción y demostración ya eliminados por Foucault.

En este orden de ideas vuelvo a citar a Gadamer (1997) en su definición de lo bello, esta vez refiriéndose a la poesía: "Llamamos bello a algo que está justificado por su propio ser y no conoce ninguna instancia fuera de sí mismo ante la que tuviera que justificarse" así podríamos deducir que lo más importante en la obra es aquello que calla, porque carece de esta necesidad de autojustificarse, tal justificación entendida desde los límites ya expuestos en nuestra forma de conocer. En cambio, si nos remitimos a la palabra, que existe también independiente de lo que pensemos, la obra de Magritte es una imagen de las palabras mismas, lo que leo me lleva a discernir entre lo que veo y lo que vivo. Aunque estas palabras estén colocadas fuera de la pipa, no dan una autoridad aparente para negar la representación de lo que observo, pero tampoco la imagen de la pipa lo afirmaría. Para Foucault, en otras palabras, la pipa desaparece, vemos en la obra que no existe tal pipa y eso, precisamente, es un fenómeno paradójico de la relación limítrofe de nuestro lenguaje con lo que este parece querer decir.

Pensar en cómo hemos hecho esta apropiación del lenguaje, esta emancipación de lo espiritual en el juicio, es lo que claramente nos controla y nos aleja de lo que vivimos, de nuestra lectura real del mundo. ${ }^{10}$ Lo que además nos lleva a dar solo un valor histórico a los nominalismos, teniendo en cuenta que son útiles, al ser estos una construcción del hombre.

\footnotetext{
${ }^{9}$ Es necesario asumir el primer lenguaje, el lenguaje discursivo, como uno y el mismo lenguaje ordinario para Wittgenstein. Luego, aceptar que la misma limitación del lenguaje nos lleva a considerar algún lenguaje fuera del discurso, lo que he presentado aquí como el lenguaje que se refiere al arte.

${ }^{10}$ Retomo aquí las primeras experiencias con los objetos, las cuales anteceden al lenguaje.
}

El lenguaje que utilizamos es, entonces, un lenguaje que limita y por tanto se queda pobre respecto a nuestras capacidades de percepción y experiencias sensibles. Aunque consideráramos la lógica como base de todo lo que hemos descubierto, nuestra manera de transformar las ideas en el discurso se aleja de la esencia de nuestro interactuar e interaccionar con todo lo que nos rodea, esto es, lo que se infiere de las experiencias reales, pero al final se halla solamente representado.

Podría concluir que lo que demuestra una imposibilidad en el lenguaje que conocemos se atribuye a la facultad del conocer y no al lenguaje mismo y, a riesgo de parecer reiterativo, es claro que esto se evidencia en lo que la obra de arte finalmente solo nos muestra. Nuestra facultad de conocimiento absoluto por medio de las palabras es inalcanzable, y el lenguaje no escapa al error porque este es, en esencia, producto de la contingencia, situación que como ya lo hice notar nonos dará una última interpretación de lo que hemos experimentado delante de una obra de arte, entonces, parece evidente que ello solo podremos saberlo cuando lo volvamos a experimentar.

\section{Referencias Bibliográficas}

Foucault, M. (1989). Esto no es una pipa, ensayo sobre Magritte [2 $\left.{ }^{\mathrm{a}} \mathrm{ed}.\right]$. Barcelona: Editorial Anagrama.

Gadamer, H.-G. (1997). La actualidad de lo bello. En H.-G. Gadamer, La justificación del arte (pp.29-66). Barcelona: Editorial Paidós.

Gadamer, H.-G. (1998). El texto "eminente" y su verdad.En H.-G. Gadamer, Arte, verdad y palabra (prólogo de Gerard Vilar, pp. 95-109). Barcelona: Editorial Paidós.

Sthendal (1998). Nápoles y Florencia: un viaje de Milán a Reggio. Valencia: Editorial Pre-Textos.

Wittgenstein, L. (1997). Ocasiones filosóficas. Madrid: Ed. Cátedra.

Wittgenstein, L. (2010). Tractatus logico-philosophicus. Madrid: Alianza Editorial.

Laura Mariana Moreno Segura. Estudiante de cuarto semestre de la Licenciatura en Artes Visuales de la Universidad Pedagógica Nacional. Participó en la cátedra Cinemateca 2013, "Éticas, políticas y estéticas del cine colombiano", en el Encuentro Nacional de Educación Artística realizado en Bogotá del 26 al 29 de agosto de 2013; y en el I Coloquio Interno de la Licenciatura en Filosofía, realizado del 22 al 26 de abril de 2013 con la ponencia titulada: "La obra de arte no dice nada".

dav_lmmorenos336@pedagogica.edu.co

Artículo recibido en abril de 2013 y aceptado en agosto de 2013 